\title{
Expression of nuclear survivin in normal skin and squamous cell carcinoma: a possible role in tumour invasion
}

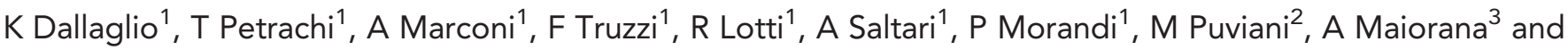 \\ C Pincelli*,1 \\ ${ }^{1}$ Laboratory of Cutaneous Biology, Institute of Dermatology, University of Modena and Reggio Emilia, Via del Pozzo 71, \\ 41124 Modena, Italy; ${ }^{2}$ Department of Dermatology and Dermatological Surgery, Nuovo Ospedale Civile di Sassuolo, \\ Via Francesco Ruini 2, 41049 Sassuolo Modena, Italy and ${ }^{3}$ Department of Laboratories and Pathologic Anatomy, University of \\ Modena and Reggio Emilia, Via del Pozzo 71, 41124 Modena, Italy
}

Background: Survivin is detected in few adult normal cells and it is highly expressed in cancer. Nuclear survivin facilitates cell cycle entry, whereas the mitochondrial pool protects cells from apoptosis. Survivin is overexpressed in keratinocyte stem cells (KSCs) and protects them from apoptosis.

Methods: As KSCs are at the origin of squamous cell carcinoma (SCC), we evaluated survivin expression in normal and cancerous skin in vivo by immunohistochemistry and western blotting. $\mathrm{HaCaT}$ cells overexpressing survivin and wound-healing assay are used. Analysis of variance and Student's T-tests are used for statistical analysis.

Results: Survivin is localised in both the cytoplasm and nucleus of normal adult and young keratinocytes. Nuclear survivin is detected in one every 10 of 11 basal keratinocytes. When present in suprabasal cells, nuclear survivin is coexpressed with K10 but not with K15 or p75-neurotrophin receptor (p75NTR), a transit amplifying cell marker. Nuclear, but not cytoplasmic, survivin expression markedly increases in actinic keratosis and in SCC in situ, as compared with normal epidermis, and it is highest in poorly differentiated SCC. In SCC tumours, nuclear survivin-positive cells are mainly K10/p75NTR-negative and K15-positive. In poorly differentiated tumours, survivin mostly localises in the deep infiltrating areas. When overexpressed in keratinocytes, survivin increases cell migration.

Conclusion: High survivin expression and the subcellular localisation of survivin correlate with keratinocyte differentiation and are associated with undifferentiated and more invasive SCC phenotype.

Cutaneous squamous cell carcinoma (cSCC) is the second most common non-melanoma skin cancer, with increasing incidence in the past decades (Martorell-Calatayud et al, 2013). Because the number of deaths caused by cSCC is now similar to that counted for melanoma (Martorell-Calatayud et al, 2013), a deepened knowledge of cSCC biology, including the factors determining CSCC aggressiveness, is necessary. According to the new American Joint Committee on Cancer (AJCC), the degree of cSCC differentiation determines, along with several other factors, the tumour-specific stage of the disease (Farasat et al, 2011). In normal skin, epidermal differentiation is a finely regulated physiologic process, consisting of a progressive maturation of undifferentiated cells from the basal layers to the skin surface (Pincelli and Marconi, 2010). Although still under debate (Boehnke et al, 2012), evidence suggests that cSCC seems to originate from a mutated keratinocyte stem cell (KSC) located in the basal layer of interfollicular epidermis (Morris, 2000).

Survivin, an IAP protein with the ability to both block apoptosis and regulate cell cycle, almost exclusively, marks a population of 
normal human keratinocytes with stem cell characteristics (Marconi et al, 2007). Interestingly, survivin functions are tightly associated with its intracellular localisation (Colnaghi et al, 2006). In particular, the nuclear pool of survivin orchestrates cell division, whereas mitochondrial survivin protects cells from apoptosis by preventing caspase activation, when released to the cytoplasm (Dohi et al, 2004). Finally, survivin can also shift from the nucleus to the cytoplasm, where its role is not completely defined (reviewed in Dallaglio et al, 2012), yet being associated with the antiapoptotic function (Chan et al, 2010), as previously shown for the mitochondrial pool (Dohi et al, 2004). Survivin is mainly nuclear in cultured KSC, where it correlates with high proliferative activity, whereas it is located in the cytoplasm of transit amplifying (TA) cells (Marconi et al, 2007). In addition, survivin is expressed in the cytoplasm of basal keratinocytes from normal human skin in vivo (Marconi et al, 2007). Although survivin is expressed more in precancerous and cancerous skin than in normal skin (Chiodino et al, 1999; Lo Muzio et al, 2001; Bowen et al, 2004; Park et al, 2004), a complete understanding of survivin expression in normal, precancerous and cancerous lesions, with particular regard to the differentiation stage of the disease, is still lacking. In this work, we analysed the correlation between survivin intracellular localisation and the differentiative state of keratinocytes and SCC cells. We present evidence that survivin is expressed not only in the cytoplasm but also in the nucleus of basal and suprabasal keratinocytes. Poorly differentiated cSCC express highest levels of nuclear survivin (N-surv), whereas its expression decreases in actinic keratosis $(\mathrm{AK})$ and in more differentiated cSCC. Finally, $\mathrm{N}$-surv localises mainly in the deepest nests of cSCC, whereas its overexpression increases keratinocyte migration.

\section{MATERIALS AND METHODS}

Clinical samples. Skin samples from normal (adult or young) individuals, AK, in situ SCC and well-differentiated (WD), moderately differentiated (MD) or poorly differentiated (PD) cSCCs were obtained from patients recruited at the Policlinico of Modena and at the Nuovo Ospedale Civile di Sassuolo (Italy). The Declaration of Helsinki protocols were followed and patients signed the informed consent from the Department of Dermatology and Dermatological Surgery, approved by the Modena Commission of Ethics (protocol no. 184/10). Inclusion criteria for lesional skin samples were the presence of the lesion in photo-exposed areas of the body, patients aged between 50 and 90 years old, no previous treatment for SCC, lesion size $\geqslant 1 \mathrm{~cm}$ in diameter. Half of each sample was fixed for pathological confirmation. Only samples following the inclusion criteria were analysed. The number of samples tested are reported in Figure 3A. For normal human skin analysis, skin biopsies were obtained from sun-exposed areas of the trunk either from young (0-30 years old) individuals or adult/old (30-90 years old) individuals.

Western blot analysis. Normal human keratinocytes from both adult and young individuals, cSCC-derived keratinocytes and SCC13 cells, were lysed with Chemicon Nucelar Extraction kit (Chemicon International Inc., Temecula, CA, USA) to perform subcellular fractionation that separates nuclear and cytosolic components. Alternatively, HaCaT cells were lysed in RIPA buffer. Western blotting was performed as described previously (Dallaglio et al, 2013). Nitrocellulose membranes (Millipore, Billerica, MA, USA) were incubated with rabbit polyclonal anti-human survivin antibody ( $1: 1000$; Abcam, Cambridge, UK), goat polyclonal antienolase antibody ( $1: 1000$; Santa Cruz Biotechnology, Santa Cruz, CA, USA) as a cytosolic positive control or with anti- $\beta$-actin, mouse monoclonal antibody (1:5000; Sigma, St Louis, MO, USA) as a loading control. The band intensities were quantitatively determined using ImageJ software (NIH, Bethesda, MD, USA), and the intensity of protein levels was normalised to $\beta$-actin expression.

Primary cells and cell lines. The spontaneously transformed keratinocyte line HaCaT was kindly provided by Dr N Fusenig (DKFZ, Heidelberg, Germany) and cultured in Dulbecco's modified Eagle's medium supplemented with $10 \%$ foetal bovine serum at $37^{\circ} \mathrm{C}$ and $5 \% \mathrm{CO}_{2}$ as described (Boukamp et al, 1988). Squamous cell carcinoma 13 cell line (a kind gift from Fiona Watt, King's College, London, UK) was cultured in the presence of mitomycin-C-treated J2-3T3 feeder cells as described previously (Gandarillas and Watt, 1997). Total keratinocytes were obtained from normal human epidermis derived from young or adult individuals as described previously (Marconi et al, 2004). Briefly, the epidermis was separated from the dermis with forceps, after overnight incubation with dispase (Roche, Mannheim, Germany). After treatment of the epidermal sheet with trypsin $0.25 \% /$ EDTA $0.02 \%$, total keratinocytes were isolated, then washed in PBS and finally lysed for western blotting analysis.

Primary cSCC cells were obtained from surgical excision of the affected areas. Cutaneous squamous cell carcinoma keratinocytes were obtained by mincing total skin, followed by incubation for $2 \mathrm{~h}$ with dispase (Roche) and Ultrapure Collagenase Type III (Sigma) at $37^{\circ} \mathrm{C}$. The remaining tissue was incubated at $37^{\circ} \mathrm{C}$ for $20 \mathrm{~min}$ with trypsin $0.05 \% /$ EDTA $0.02 \%$. Once total keratinocytes were extracted, cells were plated on mitomycin-C treated J2-3T3 feeder layer at $10^{5}$ to $3 \times 10^{5}$ per $100 \mathrm{~mm}$ dish density, in the keratinocyte medium (Purdie et al, 2011).

Infection of HaCaT cells. A total of $16 \times 10^{3}$ per $\mathrm{cm}^{2} \mathrm{HaCaT}$ cells were plated, and $24 \mathrm{~h}$ later, infected two times with conditioned medium containing retrovirus for EGFP (enhanced green fluorescent protein) or survivin-EGFP (codified by pcz-CFG5.1-EGFP or pcz-CFG5.1-survivin-EGFP retroviral vector, a kind gift from Achim Temme, Technische Universitat, Dresden, Germany) and polybrene to a final concentration of $0.8 \mu \mathrm{g} \mathrm{ml}^{-1}$. Thereafter, the culture medium was changed, cells were trypsinised and the number of EGFP-positive cells was controlled by flow cytometry, performed as described previously (Temme et al, 2003). Only if the infection efficiency was at least $80 \%$, HaCaT cells were used for the experiments.

Scratch wound-healing/migration assay. HaCaT cells were grown up to $90 \%$ confluency in six-well tissue culture plates and treated with mitomycin C. Scratches were then made on the plates using a sterile $200 \mu \mathrm{l}$ plastic tip. The plates were then carefully washed two times with serum-free medium and fresh culture medium was gently added onto the plates. The plates were then monitored over the next $48 \mathrm{~h}$. Photographs were taken on six different areas to monitor gap closure. The result of each experiment was expressed as the \% of the mean of migrated cells from six different areas.

Immunohistochemical analysis. Immunohistochemistry for survivin analysis was performed as follows. Formalin-fixed, paraffin-embedded tissue was cut into 4 - $\mu \mathrm{m}$ sections and mounted on poly-L-lysine-coated slides. After deparaffinisation and rehydration, the slides were boiled in citrate buffer ( $\mathrm{pH} \mathrm{6)}$ for $30 \mathrm{~min}$. Then, a primary rabbit polyclonal antibody against $\mathrm{N}$-surv and cytoplasmic survivin (1:300; Abcam) was used in a biotin-free system where antibody-HRP-labeled antibody complex is visualised using 3,3'-diaminobenzidine (ultraView Universal DAB detection kit; Ventana, Oro Valley, AZ, USA). Negative controls were run simultaneously by omitting primary antibody while incubating with buffer. Double staining was continued by incubating the slides with either $2 \mu \mathrm{g} \mathrm{ml}^{-1}$ mouse anti-p75neurotrophin receptor (p75NTR) antibody (Lab Vision Corporation, Neomarkers, Fremont, CA, USA) or rabbit monoclonal antiK10 antibody ( $1: 100$; Epitomics Inc., Burlingame, CA, USA) or 
rabbit monoclonal anti-K15 (1:100; Epitomics Inc.) for $1 \mathrm{~h}$. Slides were then incubated with secondary antibody anti-mouse $(1: 20$; Dako, Glostrup, Denmark) or anti-rabbit biotinylated antibody (1:300; DakoCytomation, Glostrup, Denmark) for $45 \mathrm{~min}$ at room temperature, followed by incubation with anti-mouse alkaline phosphatase complex for $30 \mathrm{~min}$ (1:20; Dako). After three washes for $5 \mathrm{~min}$ in PBS, the slides were stained with Fast blue (Sigma) and lightly counterstained with Harris haematoxylin (Sigma) for $20 \mathrm{~min}$. Staining for $\mathrm{N}$-surv in each nucleus was classified as positive or negative, based on the evaluations of two independent observers, in a blinded manner. Four hundred cells were examined per field at $\times 40$ magnification.

To estimate survivin expression, two different evaluation methods have been used:

i Three to five pictures, representative of the entire lesion, independently from survivin-positive staining, were taken. Both positive and negative areas for survivin expression were included in the calculation.

ii Three to five pictures of areas displaying nuclear staining of survivin were taken. Only areas with positive staining were included in the evaluation.

In both cases, the number of stained N-surv-positive cells (both strongly, moderately and weakly stained) were counted and normalised to the total number of haematoxylin-positive nuclei. The mean percentage of positive cells was determined. To evaluate the frequency of positive nuclei relative to basal cells, 300 basal cells were examined in at least 4 areas at $\times 40$ magnification. The number of suprabasal and basal-positive nuclei was counted and expressed as the number of positive nuclei/basal cell. This was performed on at least three different sections and the results were reported as a mean. Cytoplasmic survivin and $\mathrm{N}$-surv expression was scored semiquantitatively as strong $(+++)$, moderate $(++)$, weak $(+)$ or negative $(-)$. Survivin distribution in lesional skin has been indicated as localised, when restricted to precise areas of the lesion, or homogeneous, when equally distributed.

Statistical analysis. Statistical analysis was performed first by ANOVA, and, in case of significance $(P<0.05)$, also by post hoc comparison using Student's $t$-test.

\section{RESULTS}

$\mathrm{N}$-surv expression in normal human epidermis. Because survivin is overexpressed in KSC (Marconi et al, 2007; Altieri, 2008), and stem cell activity is reduced during ageing in several tissues (Martin et al, 1998; Wagner et al, 2009), we evaluated survivin expression in the skin from young (0-30 years old) and adult/old (30-90 years old) individuals. Nuclear and cytoplasmic survivin was expressed in normal human epidermis from both young and adult subjects. Although cytoplasmic survivin was present only in basal cells, N-surv-positive cells were located in the basal and immediately suprabasal epidermal layers (Figure $1 \mathrm{~A}$ and Table 1). In addition, survivin was detected in both nuclear and cytosolic extracts, although to a greater extent in the latter fraction in both young and adult epidermis (Figure 1B). The number of $\mathrm{N}$-surv-positive nuclei was higher in young than in adult specimens (Table 1). Moreover, N-surv-positive nuclei were more frequent in basal keratinocytes from young than from adult epidermis. We then evaluated the differentiation and stem-associated markers in normal epidermis through a double staining with survivin (Figure 2). Most N-surv-positive cells expressed K10, an early differentiation marker in human epidermis (Fuchs and Green, 1980), in both young and adult specimens. On the other hand, N-surv-positive cells failed to express either the putative stem cell marker K15 (Webb et al, 2004) or p75NTR that is mainly localised in TA cells (Truzzi et al, 2011).

\begin{tabular}{|c|c|c|c|c|c|}
\hline \multicolumn{4}{|c|}{ Survivin expression } & \multicolumn{2}{|c|}{$\begin{array}{l}\text { Positive nuclei } \\
\text { frequency }\end{array}$} \\
\hline Case & Cytoplasmic & $\begin{array}{l}\text { Nuclear } \\
\text { (\%) }\end{array}$ & $\begin{array}{c}\text { Nuclear } \\
\text { mean } \\
(\%)\end{array}$ & Age & $\begin{array}{c}\text { Pos. nuclei/ } \\
\text { basal cells } \\
\text { mean }\end{array}$ \\
\hline Young $2 \mathrm{YO}$ & ++ & $\begin{array}{c}+++ \\
(7.3)\end{array}$ & Young: 5.8 & Young & $1 / 6.96$ \\
\hline Young 6 YO & ++ & $++(4.3)$ & & Adult & $1 / 11.9$ \\
\hline Young 4 YO & ++ & $++(5.8)$ & & & $\begin{array}{c}\text { Total: 1/ } \\
9.43\end{array}$ \\
\hline Adult $35 \mathrm{YO}$ & ++ & $+(1.9)$ & Adult: 2.2 & & \\
\hline Adult 46 YO & $+1-$ & $+(1.3)$ & & & \\
\hline \multirow[t]{2}{*}{ Adult 56 YO } & ++ & $+(3.5)$ & & & \\
\hline & & & Total: 4 & & \\
\hline
\end{tabular}

A

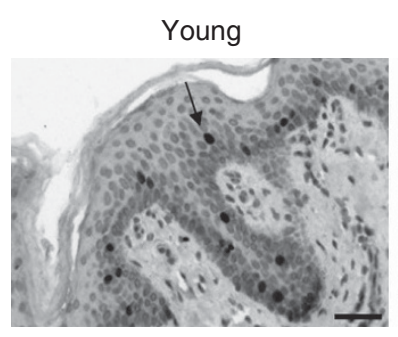

Adult

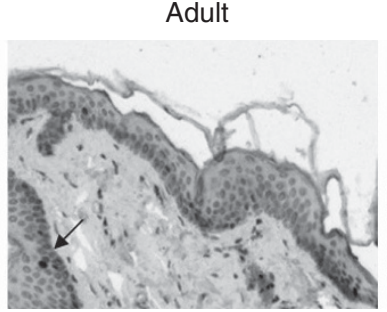

Neg CNTRL

B

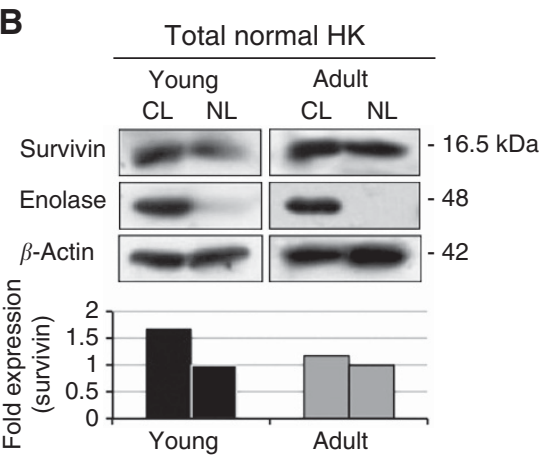

Figure 1. Expression of nuclear and cytoplasmic survivin in normal epidermis from young and adult individuals. (A) Immunohistochemical analysis of survivin expression in normal human skin from young and adult individuals. Nuclear survivin is indicated by arrows (bars $=70 \mu \mathrm{m})$. (B) Total keratinocytes from young or adult healthy individuals were isolated, as described in Materials and Methods. Nuclear lysates (NL) and cytosolic lysates (CL) were made and western blot analysis was performed. Enolase was used as a positive control (CNTRL) for cytosolic extracts; $\beta$-actin was used as a loading control. Band intensity was quantified using Image $\mathrm{J}$ software. 


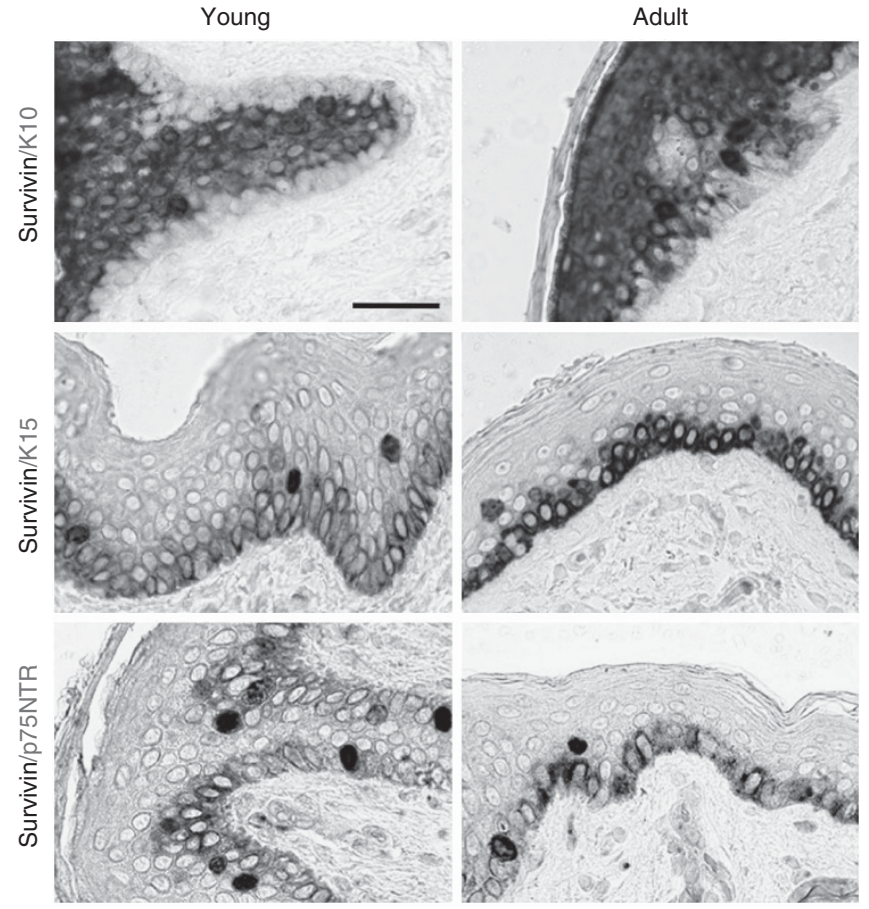

Figure 2. Survivin and differentiation-related markers in healthy skin. Double staining of representative young and adult skin for survivin and either K10, K15 or p75NTR. Fast blue was used as chromogen for K10, K15 and P75NTR, whereas 3,3'-diaminobenzidine was used for survivin. Representative data of three experiments are shown. Bars $=70 \mu \mathrm{m}$.

$\mathrm{N}$-surv is overexpressed in AK, in situ SCC and cSCC. To better clarify the expression pattern of survivin in precancerous and cancerous skin, AK, in situ SCC and cSCC were included in the study, and cSCC were divided into three main categories based on the differentiation grade of the tumour (WD, MD and PD). As $\mathrm{N}$-surv is expressed more in young epidermis, and cSCC lesions preferentially occur in adult and old individuals, healthy skin from adult donors was used as a control. Moreover, being UVB, one of the most common risk factor associated with cSCC, only skin biopsies from normal sun-exposed areas were included in the study. Unlike healthy epidermis, cytoplasmic survivin was absent in precancerous and cancerous lesions. On the other hand, N-survpositive cells were more abundant in AK and in situ SCC than in normal epidermis. Moreover, in normal epidermis, N-surv-positive cells were mostly located in basal or in immediately suprabasal keratinocytes, whereas in precancerous and cancerous lesions, they were expressed only at the suprabasal level, including the spinous and the granular layers (Figure $3 \mathrm{~B}$ and $\mathrm{E}$ ). In cSCC, N-surv was expressed more in poorly differentiated tumours than in more differentiated tumours according to ANOVA and Student's $t$-test (Figure 3C, E and F). To confirm survivin overexpression in the nucleus of cSCC cells, we obtained nuclear and cytosolic extracts from SCC13 (Rheinwald and Beckett, 1981) and from cultured keratinocytes isolated from primary cSCCs (see Materials and Methods section). Both cSCC cell types expressed more N-surv than cytoplasmic survivin (Figure 3D).

To compare survivin expression in the different skin lesions, we calculated the percentage of positive nuclei on the total number of cells counted in each field. While N-surv was only scattered in some areas of $\mathrm{AK}, \mathrm{MD}$ and $\mathrm{WD}$ CSCC, it was diffuse and homogeneously distributed in situ and in PD cSCC (Figure 3C). To take into account tumour heterogeneity in the evaluation of survivin distribution in the different lesions, we compared the results obtained by using two different cell count approaches (see Materials and Methods). In Figure 3E and Table 2, the percentage of positive nuclei was calculated by evaluating $\mathrm{N}$-surv expression in the whole lesion. This was performed by analysing six distant fields chosen randomly in the lesion area (Method I). By this method, $\mathrm{N}$-surv was significantly more expressed in $\mathrm{AK}$ and cSCCs than in healthy skin. In addition, N-surv was more expressed in in situ cSCCs than in WD and MD cSCC, whereas it was highest in PD tumours. We then analysed N-surv expression in the same section by using an alternative method based on the calculation of the percentage of N-surv-positive cells only in areas in which the positive nuclei were detectable (Method II). In Figure $3 \mathrm{C}$ and Table 3 , there is an overall increase in the percentage of positive cells when compared with that obtained by Method I. Yet, N-surv-positive cells were still significantly higher in in situ cSCC and PD tumours than in WD and MD cSCC. On the other hand, by Method II, no significant difference was observed between in situ and total cSCC. We can conclude that, depending on the areas included in the cell count, the percentage of N-surv-positive cells markedly changes resulting in a significant difference between in situ and cSCC only when a global evaluation of the lesion is performed.

$\mathrm{N}$-surv is predominantly expressed in less differentiated cSCC cells. To better correlate the expression pattern of survivin to the differentiative state of tumour, we performed a double staining for survivin and either K10, K15 or p75NTR (Figure 4). In both AK and in situ SCC, N-surv-positive cells expressed K10, as observed in the normal epidermis (Figure 2). By contrast, in cSCC lesions, K10 was irregularly distributed in both basal and suprabasal cells, and was not expressed in the majority of N-surv-positive cells, this being more evident in PD cSCC. We detected K15 in all cSCC lesions, although at different levels. In AK and in in situ SCC, K15 was mainly detected in basal keratinocytes, and it was not expressed in N-surv-positive cells. On the other hand, in cSCC lesions, K15 was distributed in all epidermal layers and in most $\mathrm{N}$-surv-positive cells, with no apparent difference among tumour categories. Finally, p75NTR, which is irregularly expressed in normal basal keratinocytes (Figure 2; Truzzi et al, 2011), was absent in AK and in all cSCC, except for isolated positive cells in a few cSCC (see arrow) that failed to express N-surv (Figure 4). We concluded that survivin and p75NTR do not colocalise, as previously shown in the normal epidermis (Figure 2).

$\mathrm{N}$-surv is expressed in invasive areas of cSCC and sustains cell migration. Finally, we analysed survivin distribution in different areas of PD cSCC. Survivin was mainly cytoplasmic and was located in basal keratinocytes of non-lesional epidermis, far from the tumour. On the other hand, in areas of apparently healthy skin, close to the tumour, survivin shifted to the nucleus and it was also expressed in immediately suprabasal keratinocytes. In the intraepidermal tumour, the number of $\mathrm{N}$-surv-positive cells increased, to eventually become more numerous in the deepest cell nests of infiltrating cSCC areas (Figure 5A). Because survivin overexpression in cancer infiltrates often correlates with tumour metastasis (Lo Muzio et al, 2001), and some lines of evidence suggests an involvement of survivin in cell migration (McKenzie et al, 2010), we wanted to address whether survivin overexpression in deeper cell nests is associated with the potential of keratinocytes to migrate. HaCaT cells overexpressing survivin migrated to a significantly greater extent as compared with mock cells. (Figure 5B). These data seem to suggest that survivin has an active role in cSCC cell invasion.

\section{DISCUSSION}

Survivin is a key molecule for the maintenance of epidermal homeostasis (Marconi et al, 2007; Dallaglio et al, 2009). Yet, its 
A

\begin{tabular}{lc}
\multicolumn{1}{c}{ Lesion type } & No. \\
\hline AK & 24 \\
\hline In situ SCC & 18 \\
\hline WD SCC & 16 \\
MD SCC & 9 \\
PD SCC & 4 \\
\hline
\end{tabular}

D
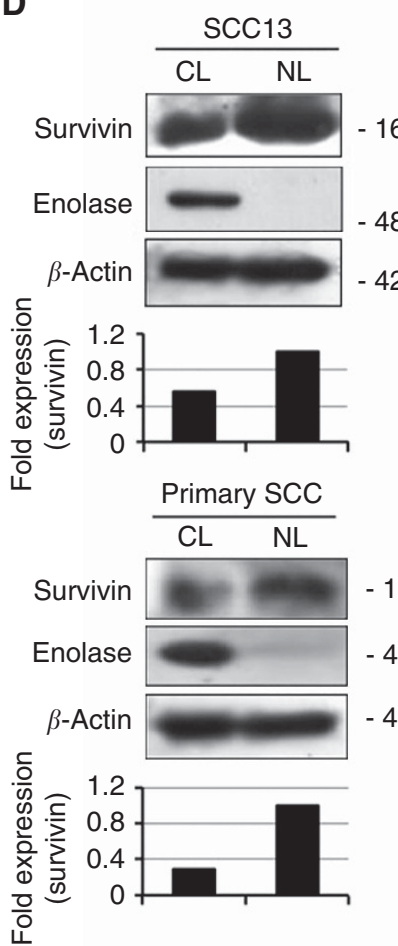

$16.5 \mathrm{kDa}$

$-48$

$-42$
B Adult healthy skin
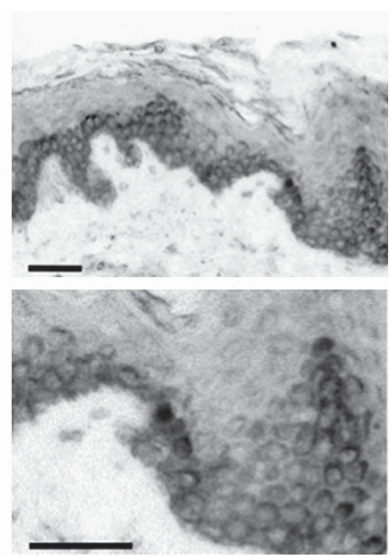

C

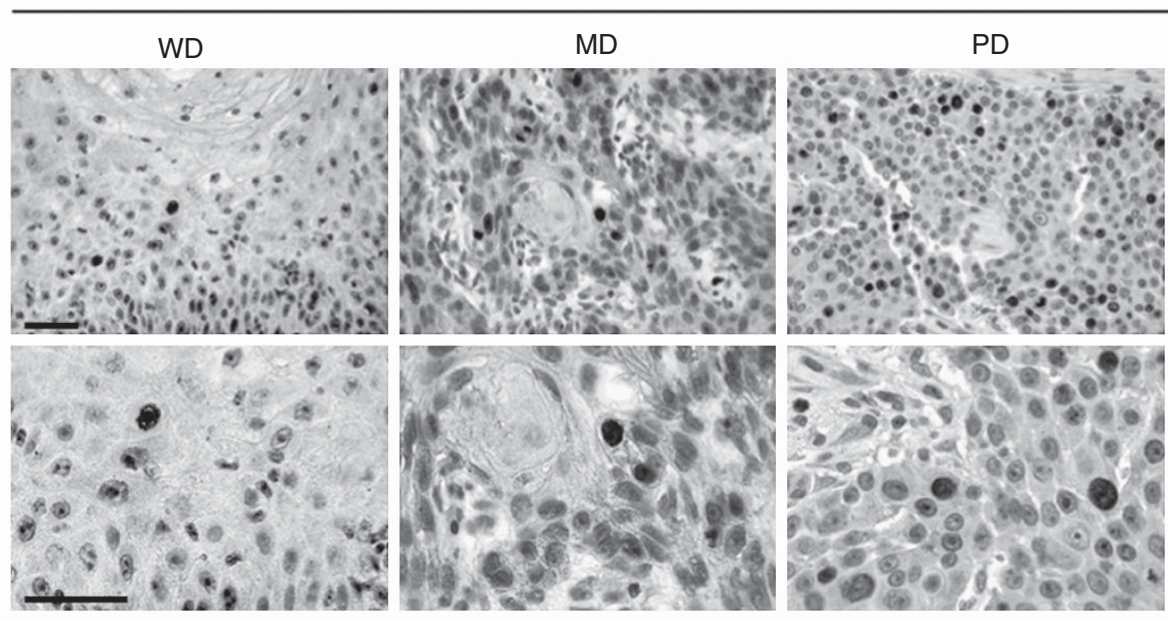

Actinic Keratosis
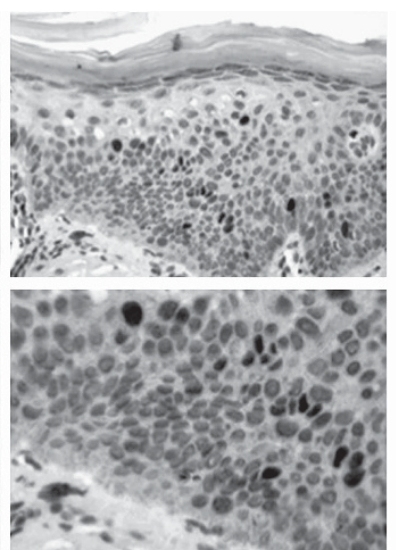

Cutaneous SCC
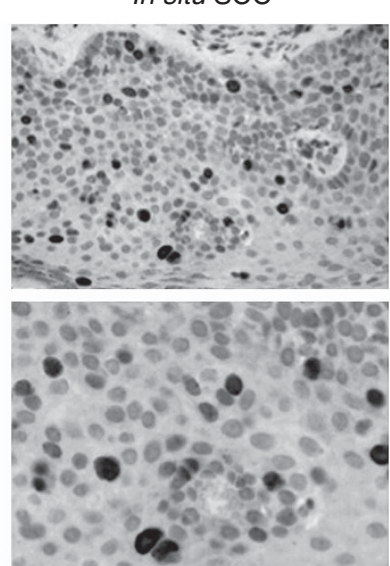

F

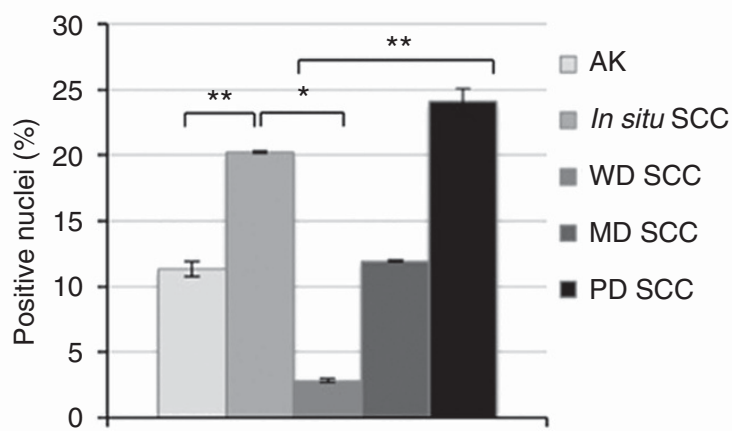

Figure 3. Expression of N-surv in AK, in situ SCC and CSCC. (A) Number of cases of AK, in situ SCC and cSCC analysed for survivin expression by immunohistochemistry. Cutaneous SCC are divided into WD, MD and PD tumours (see Materials and Methods). (B) Representative immunohistochemical staining of adult healthy skin, AK and in situ SCC for survivin expression (bars $=70 \mu \mathrm{m}$ ). (C) Representative immunohistochemical staining of WD, MD and PD cSCC for survivin expression (bars $=70 \mu \mathrm{m}$ ). (D) Squamous cell carcinoma 13 cells and total keratinocytes from primary cutaneous SCC were isolated as described in Materials and Methods. NL and CL were obtained and western blot analysis was performed. Enolase was used as a positive control for cytosolic extracts, whereas $\beta$-actin was used as a loading control. Band intensity was quantified using Image J software. (E) Quantification of N-surv-positive cells in lesional and healthy skin by using a 'whole-lesion evaluation' approach (Method I, described in Materials and Methods). ${ }^{*} P<0.05$; ${ }^{* \star} P<0.01$. (F) Quantification of N-surv-positive cells in lesional skin by evaluating areas of the lesions in which survivin staining is positive (Method II, described in Materials and Methods). ${ }^{\star} P<0.05$; ${ }^{\star \star} P<0.01$.

expression in normal human epidermis of adult individuals has been controversial. Some reports show that survivin is absent in the normal epidermis (reviewed in Dallaglio et al, 2012), whereas others have recently confirmed its presence in basal keratinocytes (Marconi et al, 2007). Nuclear survivin has been detected in hyperproliferative skin conditions, such as psoriasis and cancer (Bowen et al, 2004; Abdou and Hanout, 2008), whereas little is known on its expression in the normal epidermis. In this study, we show that the nuclear pool of survivin is expressed in the normal human epidermis and may identify early-differentiating 


\begin{tabular}{|c|c|c|c|c|}
\hline Specimens & $\begin{array}{c}\text { Nuclear } \\
\text { survivin } \\
\text { expression } \\
(\%)\end{array}$ & Intensity & $\begin{array}{c}\text { Cytoplasmic } \\
\text { surv. exp. }\end{array}$ & Distribution \\
\hline $\begin{array}{l}\text { Healthy } \\
\text { adult skin }\end{array}$ & $2.7 \pm 0.01$ & + & + & I \\
\hline$A K$ & $4.23 \pm 0.03$ & +++ & - & Localised \\
\hline In situ SCC & $8.41 \pm 0.04$ & +++ & - & Homogeneous \\
\hline WD SCC & $3.36 \pm 0.01$ & +++ & - & Localised \\
\hline MD SCC & $4.04 \pm 0.03$ & +++ & - & Localised \\
\hline PD SCC & $9.01 \pm 0.04$ & +++ & - & Homogeneous \\
\hline Total SCC & $5.47 \pm 0.03$ & +++ & - & $\begin{array}{c}\text { Localised/ } \\
\text { homogeneous }\end{array}$ \\
\hline
\end{tabular}

Abbreviations: $\quad A K=$ actinic keratosis; $\quad \mathrm{CSCC}=$ cutaneous squamous cell carcinoma; $\mathrm{MD}=$ moderately differentiated; $\mathrm{PD}=$ poorly differentiated; $\mathrm{WD}=$ well differentiated .

Table 3. Nuclear survivin expression in precancerous and cancerous lesions

\begin{tabular}{|l|c|}
\hline Lesion type & Nuclear survivin expression (\%) \\
\hline AK & $11.31 \pm 0.06$ \\
\hline In situ SCC & $20.25 \pm 0.1$ \\
\hline WD SCC & $12.81 \pm 0.07$ \\
\hline MD SCC & $11.93 \pm 0.08$ \\
\hline PD SCC & $24.06 \pm 0.04$ \\
\hline & $16.27 \pm 0.07$ \\
\hline Total SCC & \\
\hline Abbreviations: AK = actinic keratosis; cSCC $=$ cutaneous squamous cell carcinoma; \\
MD=moderately differentiated; $P D=$ poorly differentiated; WD = well differentiated. \\
\hline
\end{tabular}

keratinocytes in healthy skin. In skin tumours, N-surv correlates with the differentiation grade of the lesion. The discrepancy with previous reports is probably due to differences in methodology, assay sensitivity, specificity of the antibody, variations in assay conditions or even the age of the donors. To overcome the age bias, we analysed both young and adult epidermis from healthy individuals. Higher N-surv positivity and frequency in specimens from younger individuals suggest ageing as a factor that may influence survivin expression. As $\mathrm{N}$-surv is involved cell division (Altieri, 2008), the lower levels in normal skin from older individuals is in line with the reduced proliferative activity of aged epidermis (Gilchrest, 1983). On the basis of our previous results showing survivin overexpression in the nuclei of KSC, which in turn correlates to their high proliferation index in vitro (Marconi et al, 2007), we first supposed that in vivo N-surv positive keratinocytes are proliferating cells. Similarly to the proliferation marker Ki-67 (Gilhar et al, 2004), N-surv-positive cells are more numerous in the epidermis from young individuals than from adult individuals. However, not all N-surv-expressing cells stain for Ki-67 in human cancerous skin (Bongiovanni et al, 2011). In addition, in the normal skin, Ki-67-positive cells are usually located in the basal layer of normal epidermis, whereas N-surv-positive cells are also detected in suprabasal layers. Therefore, further studies are needed to elucidate whether all of N-surv-positive cells are proliferating in healthy skin.

Keratinocyte stem cells are estimated to represent 1 to $10 \%$ of basal keratinocytes (Potten and Hendry, 1973; Li et al, 1998; reviewed in Cotsarelis et al, 1999). As in the present study $\mathrm{N}$-surv-positive cells are about $10 \%$ of total keratinocytes in the normal epidermis, one could hypothesise that these cells are KSC. However, N-surv-positive cells are located in suprabasal cell layers and express the differentiation marker K10, but not $\mathrm{K} 15$. This suggests that $\mathrm{N}$-surv is not a marker of KCS in vivo but can identify a population of cells starting the differentiation process, located at the boundary between the basal and suprabasal layers. This is consistent with the definition of TA cells, which are committed to terminal differentiation while retaining proliferative activity (Marconi et al, 2007). Moreover, number and functionality of KSC does not change with age (Stern and Bickenbach, 2007; Giangreco et al, 2008), whereas in

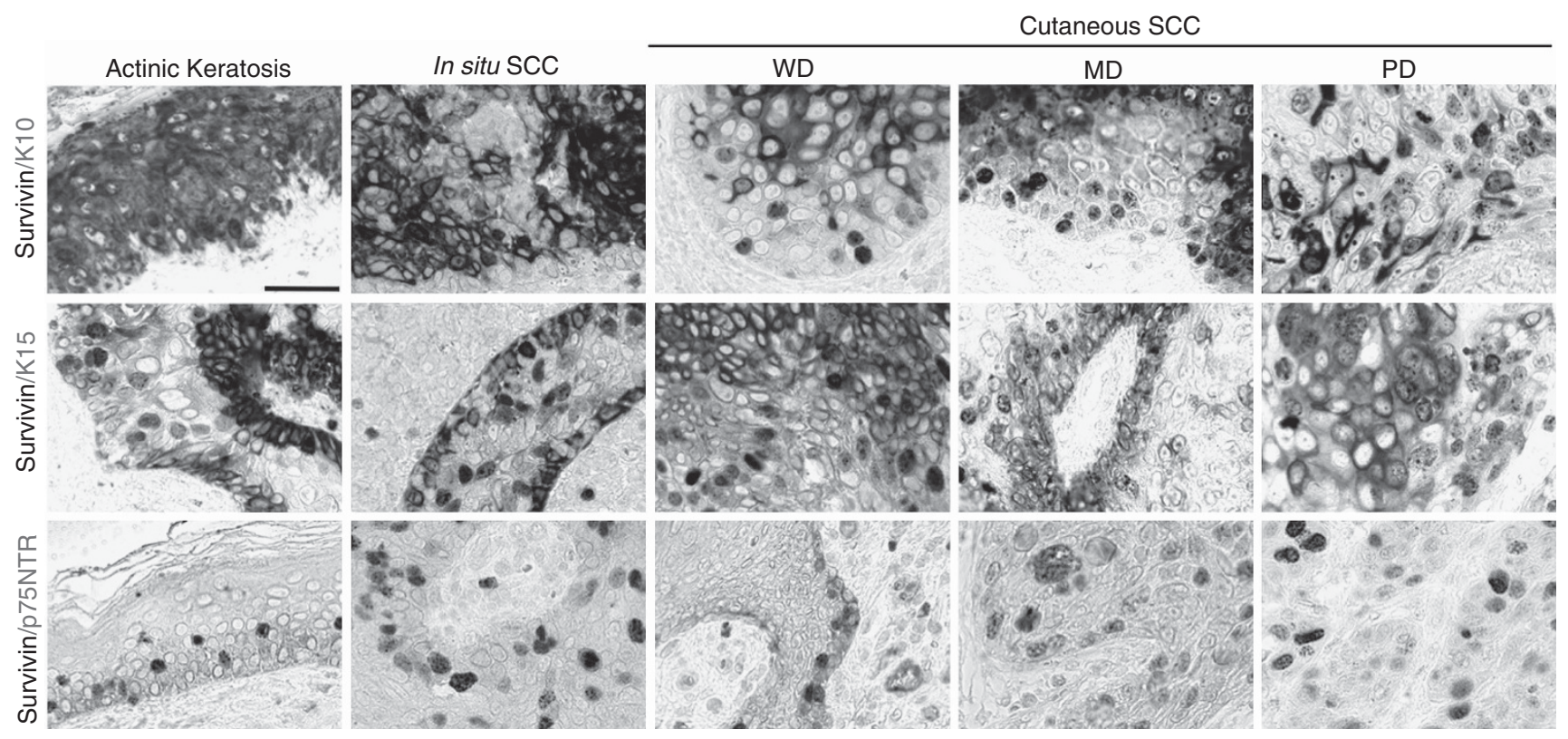

Figure 4. Survivin and differentiation-related markers in AK, in situ SCC and cSCC. Fast blue was used as chromogen for K10, K15 and p75NTR, whereas aminoethyl carbazole (AEC) was used for survivin (bars $=70 \mu \mathrm{m}$ ). Representative data of four patients per lesion type are shown. 

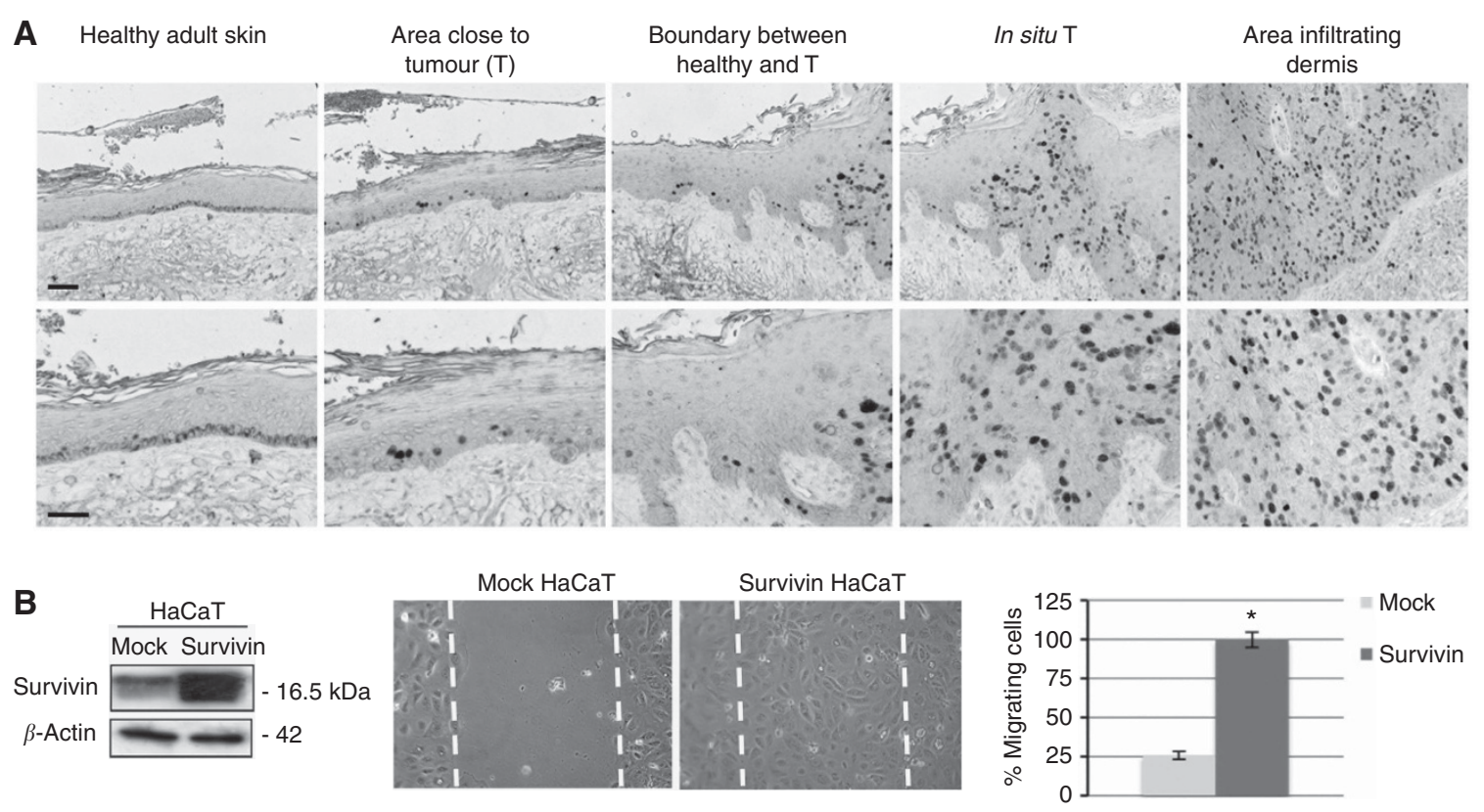

Figure 5. Nuclear survivin is overexpressed in invasive SCC and sustains keratinocyte migration. (A) Immunohistochemical analysis of survivin expression in different areas of a representative PD SCC (bars $=70 \mu \mathrm{m}$ ). (B) Mock or survivin-overexpressing HaCaT cells (confirmed by western blotting) was scratched for migration assay. Cells were then cultured for $48 \mathrm{~h}$ and migrated cells into the scraped area were observed. Six areas were counted and expressed as the mean of cell migrated per area. Data are expressed as the mean \pm s.d. of triplicate from three different experiments. ${ }^{\star} P<0.05$.

aged epidermis, TA cells display a reduced efficiency (Charruyer et al, 2009). P75-neurotrophin receptor is overexpressed by a subpopulation of TA keratinocytes (Truzzi et al, 2011), and is involved in early keratinocyte differentiation (unpublished results). Because N-surv-positive cells do not express p75NTR, $\mathrm{N}$-surv may identify a subpopulation of TA cells, which is functionally different from the one expressing p75NTR. As $\mathrm{N}$-surv-positive cells are also located in suprabasal layers, they may represent a late TA population.

Survivin overexpression has been reported in different human cancers, including skin tumours (Altieri, 2001). We have observed a shift of survivin from the cytoplasm to the nucleus of keratinocytes in AK, in in situ SCC and in cSCC sections. Cytoplasmic survivin functions mainly as an inhibitor of apoptosis, whereas $\mathrm{N}$-surv orchestrates cell division. In skin cancers and precancerous lesions, both apoptosis and cell cycle are dysregulated. We may speculate that because of the high proliferation rate observed in these lesions, survivin is preferentially sequestered in the nucleus to help cancer cells to undergo cell division. Although the expression of survivin has been previously shown to be confined to the basal layer in $\mathrm{AK}$, similarly to normal epidermis (Park et al, 2004), we found N-surv not only in basal cells but also in suprabasal keratinocytes from AK and cSCC lesions, suggesting that the appearance of positive nuclei in suprabasal cell layers is a hallmark of disrupted epidermal homeostasis. The number of $\mathrm{N}$-surv-positive cells is equal in $\mathrm{AK}$ and $\mathrm{WD}$ or $\mathrm{MD}$ cSCC, implying that survivin is not a good marker to distinguish preneoplastic lesions from cSCC, in line with previous reports (Bongiovanni et al, 2009). Interestingly, the percentage of N-survpositive cells was significantly higher in in situ SCC as compared with $\mathrm{WD}$ and MD cSCC. On the other hand, the percentage of positive cells were comparable between PD cSCC and in situ SCC and tended to be slightly different only when calculation was performed in areas of positive survivin staining. This is probably due to the diffuse distribution of $\mathrm{N}$-surv-positive cells in both tumour types, whereas the high amount of differentiated cells in
WD and MD tumours, which fail to express survivin, contribute to the decrease in the percentage of N-surv-positive cells. This also suggests the existence in CSCC of intratumour heterogeneity, a phenomenon also occurring in other tumour types, such as breast cancer (Potts et al, 2012). Survivin expression is significantly different among cSCCs, depending on the differentiation grade, in that PD cSCC display higher levels of N-surv than MD and WD tumours. In addition, when calculation of positive nuclei was performed taking into account the entire lesion, regardless of the presence of the staining (Figure 3F), in situ SCC expressed significantly higher N-surv than total cSCC (Figure 3E), probably reflecting the lack of large differentiated, survivin-negative, areas in in situ SCC. In any event, N-surv-positive cells could represent a reliable indicator to distinguish $\mathrm{PD}$ from more differentiated tumours. In substance, survivin expression correlates with cSCC aggressiveness, in accordance with previous reports (Lo Muzio et al, 2001). Consistently, survivin is mainly localised in deeper cell nests of PD cSCC. The increased ability of survivin-overexpressing keratinocytes to migrate in wounded areas in vitro further confirms the relationship between survivin expression and CSCC cell invasiveness. This is in line with previous reports showing survivin ability to sustain cell migration in other cutaneous neoplasms such as melanoma (McKenzie et al, 2010; McKenzie and Grossman, 2012).

Unlike healthy epidermis and precancerous lesions, N-survpositive cells become partially negative for K10 and positive for $\mathrm{K} 15$ in cSCC, particularly in PD cSCCs. This may reflect the extensive dysregulation of differentiation in more aggressive tumours, which culminates also in the strong K15 expression, detected in all lesions, at variance with previous results (Abbas and Bhawan, 2011). Finally, the absence of p75NTR in cancerous lesions is in line with the proapoptotic effects of this receptor (Truzzi et al, 2011), which is also reduced in other epithelial tumours (Yuanlong et al, 2008).

Altogether, these results suggest that $\mathrm{N}$-surv marks cells that are starting the differentiation process in normal epidermis, and 
it is associated with a more aggressive phenotype in human cSCC, possibly contributing to the invasive behaviour of this tumour.

\section{ACKNOWLEDGEMENTS}

We thank Fondazione Cassa di Risparmio di Modena for partially supporting the study and Paola Manni for immunohistochemical analysis of normal and pathologic skin sections.

\section{REFERENCES}

Abbas O, Bhawan J (2011) Expression of stem cell markers nestin and cytokeratin 15 and 19 in cutaneous malignancies. J Eur Acad Dermatol Venereol 25: 311-316.

Abdou AG, Hanout HM (2008) Evaluation of survivin and NF-kappaB in psoriasis, an immunohistochemical study. J Cutan Pathol 35: 445-451.

Altieri DC (2001) The molecular basis and potential role of survivin in cancer diagnosis and therapy. Trends Mol Med 7: 542-547.

Altieri DC (2008) New wirings in the survivin networks. Oncogene 27: 6276-6284.

Boehnke K, Falkowska-Hansen B, Stark HJ, Boukamp P (2012) Stem cells of the human epidermis and their niche: composition and function in epidermal regeneration and carcinogenesis. Carcinogenesis 33: 1247-1258.

Bongiovanni L, Colombi I, Fortunato C, Della Salda L (2009) Survivin expression in canine epidermis and in canine and human cutaneous squamous cell carcinomas. Vet Dermatol 20: 369-376.

Bongiovanni L, Müller EJ, Della Salda L (2011) Survivin in skin pathologies. Exp Dermatol 20: 457-463.

Bowen AR, Hanks AN, Murphy KJ, Florell SR, Grossman D (2004) Proliferation, apoptosis, and survivin expression in keratinocytic neoplasms and hyperplasias. Am J Dermatopathol 26: 177-181.

Boukamp P, Petrussevska RT, Breitkreutz D, Hornung J, Markham A, Fusenig NE (1988) Normal keratinization in a spontaneously immortalized aneuploid human keratinocyte cell line. J Cell Biol 106: 761-771.

Chan KS, Wong CH, Huang YF, Li HY (2010) Survivin withdrawal by nuclear export failure as a physiological switch to commit cells to apoptosis. Cell Death Dis 1: e57.

Charruyer A, Barland CO, Yue L, Wessendorf HB, Lu Y, Lawrence HJ, Mancianti ML, Ghadially R (2009) Transit-amplifying cell frequency and cell cycle kinetics are altered in aged epidermis. J Invest Dermatol 129: 2574-2583.

Chiodino C, Cesinaro AM, Ottani D, Fantini F, Giannetti A, Trentini GP, Pincelli C (1999) Communication: expression of the novel inhibitor of apoptosis survivin in normal and neoplastic skin. J Invest Dermatol 113: 415-418.

Colnaghi R, Connell CM, Barrett RM, Wheatley SP (2006) Separating the anti-apoptotic and mitotic roles of survivin. J Biol Chem 281: 33450-33456.

Cotsarelis G, Kaur P, Dhouailly D, Hengge U, Bickenbach J (1999) Epithelial stem cells in the skin: definition, markers, localization and functions. Exp Dermatol 8: 80-88.

Dallaglio K, Marconi A, Pincelli C (2012) Survivin: a dual player in healthy and diseased skin. J Invest Dermatol 132: 18-27.

Dallaglio K, Marconi A, Truzzi F, Lotti R, Palazzo E, Petrachi T, Saltari A, Coppini M, Pincelli C (2013) E-FABP induces differentiation in normal human keratinocytes and modulates the differentiation process in psoriatic keratinocytes in vitro. Exp Dermatol 222: 255-261.

Dallaglio K, Palazzo E, Marconi A, Dumas M, Truzzi F, Lotti R, Bontè F, Pincelli C (2009) Endogenous survivin modulates survival and proliferation in UVB-treated human keratinocytes. Exp Dermatol 18: 464-471.

Dohi T, Beltrami E, Wall NR, Plescia J, Altieri DC (2004) Mitochondrial survivin inhibits apoptosis and promotes tumorigenesis. J Clin Invest 114: 1117-1127.
Farasat S, Yu SS, Neel VA, Nehal KS, Lardaro T, Mihm MC, Byrd DR, Balch CM, Califano JA, Chuang AY, Sharfman WH, Shah JP, Nghiem P, Otley CC, Tufaro AP, Johnson TM, Sober AJ, Liégeois NJ (2011) A new American Joint Committee on Cancer staging system for cutaneous squamous cell carcinoma: creation and rationale for inclusion of tumor (T) characteristics. J Am Acad Dermatol 64: 1051-1059.

Fuchs E, Green H (1980) Changes in keratin gene expression during terminal differentiation of the keratinocyte. Cell 19: 1033-1042.

Gandarillas A, Watt FM (1997) c-Myc promotes differentiation of human epidermal stem cells. Genes Dev 11: 2869-2882.

Giangreco A, Qin M, Pintar JE, Watt FM (2008) Epidermal stem cells are retained in vivo throughout skin aging. Aging Cell 7: 250-259.

Gilchrest BA (1983) In vitro assessment of keratinocyte aging. J Invest Dermatol 81: 184s-189s.

Gilhar A, Ullmann Y, Karry R, Shalaginov R, Assy B, Serafimovich S, Kalish RS (2004) Ageing of human epidermis: the role of apoptosis, Fas and telomerase. Br J Dermatol 150: 56-63.

Li A, Simmons PJ, Kaur P (1998) Identification and isolation of candidate human keratinocyte stem cells based on cell surface phenotype. Proc Natl Acad Sci USA 95: 3902-3907.

Lo Muzio L, Staibano S, Pannone G, Mignogna MD, Mariggiò A, Salvatore G, Chieffi P, Tramontano D, De Rosa G, Altieri DC (2001) Expression of the apoptosis inhibitor survivin in aggressive squamous cell carcinoma. Exp Mol Pathol 70: 249-254.

Marconi A, Atzei P, Panza C, Fila C, Tiberio R, Truzzi F, Wachter T, Leverkus M, Pincelli C (2004) FLICE/caspase-8 activation triggers anoikis induced by beta1-integrin blockade in human keratinocytes. J Cell Sci 117: $5815-5823$.

Marconi A, Dallaglio K, Lotti R, Vaschieri C, Truzzi F, Fantini F, Pincelli C (2007) Survivin identifies keratinocyte stem cells and is downregulated by anti-betal integrin during anoikis. Stem Cell 25: 149-155.

Martin K, Kirkwood TB, Potten CS (1998) Age changes in stem cells of murine small intestinal crypts. Exp Cell Res 241: 316-323.

Martorell-Calatayud A, Sanmartín Jimenez O, Cruz Mojarrieta J, Guillén Barona C (2013) Cutaneous squamous cell carcinoma: defining the high-risk variant. Acta Dermosifiliogr 104: 367-379.

McKenzie JA, Grossman D (2012) Role of the apoptotic and mitotic regulator survivin in melanoma. Anticancer Res 32: 397-404.

McKenzie JA, Liu T, Goodson AG, Grossman D (2010) Survivin enhances motility of melanoma cells by supporting Akt activation and \{alpha\}5 integrin upregulation. Cancer Res 70: 7927-7937.

Morris RJ (2000) Keratinocyte stem cells: targets for cutaneous carcinogens. J Clin Invest 106: 3-8.

Park HR, Min SK, Cho HD, Kim KH, Shin HS, Park YE (2004) Expression profiles of p63, p53, survivin, and hTERT in skin tumors. J Cutan Pathol 31: 544-549.

Pincelli C, Marconi A (2010) Keratinocyte stem cells: friends and foes. J Cell Physiol 225: 310-315.

Potten CS, Hendry JH (1973) Letter: clonogenic cells and stem cells in epidermis. Int J Radiat Biol Relat Stud 24: 537-540.

Potts SJ, Krueger JS, Landis ND, Eberhard DA, David Young G, Schmechel SC, Lange H (2012) Evaluating tumor heterogeneity in immunohistochemistry-stained breast cancer tissue. Lab Invest 92: 1342-1357.

Purdie KJ, Pourreyron C, South AP (2011) Isolation and culture of squamous cell carcinoma lines. Methods Mol Biol 731: 151-159.

Rheinwald JG, Beckett MA (1981) Tumorigenic keratinocyte lines requiring anchorage and fibroblast support cultures from human squamous cell carcinomas. Cancer Res 41: 1657-1663.

Stern MM, Bickenbach JR (2007) Epidermal stem cells are resistant to cellular aging. Aging Cell 6: 439-452.

Temme A, Rieger M, Reber F, Lindemann D, Weigle B, Diestelkoetter-Bachert P, Ehninger G, Tatsuka M, Terada Y, Rieber EP (2003) Localization, dynamics, and function of survivin revealed by expression of functional survivinDsRed fusion proteins in the living cell. Mol Biol Cell 14: 78-92.

Truzzi F, Marconi A, Atzei P, Panza MC, Lotti R, Dallaglio K, Tiberio R, Palazzo E, Vaschieri C, Pincelli C (2011) P75 neurotrophin receptor mediates apoptosis in transit-amplifying cells and its overexpression restores cell death in psoriatic keratinocytes. Cell Death Differ 18: 948-958. 
Wagner W, Bork S, Horn P, Krunic D, Walenda T, Diehlmann A, Benes V, Blake J, Huber FX, Eckstein V, Boukamp P, Ho AD (2009) Aging and replicative senescence have related effects on human stem and progenitor cells. PLoS One 4: e5846.

Webb A, Li A, Kaur P (2004) Location and phenotype of human adult keratinocyte stem cells of the skin. Differentiation 72: 387-395.

Yuanlong H, Haifeng J, Xiaoyin Z, Jialin S, Jie L, Li Y, Huahong X, Jiugang S, Yanglin P, Kaichun W, Jie D, Daiming F (2008) The inhibitory effect of p75 neurotrophin receptor on growth of human hepatocellular carcinoma cells. Cancer Lett 268: 110-119.

This work is published under the standard license to publish agreement. After 12 months the work will become freely available and the license terms will switch to a Creative Commons AttributionNonCommercial-Share Alike 3.0 Unported License. 